\title{
Temperature dependence of dynamic nuclear polarization and its effect on electron spin relaxation and dephasing in InAs/GaAs quantum dots
}

\author{
Jan Beyer, Yuttapoom Puttisong, Irina Buyanova, S. Suraprapapich, \\ C. W. Tu and Weimin Chen
}

\section{Linköping University Post Print}

N.B.: When citing this work, cite the original article.

Original Publication:

Jan Beyer, Yuttapoom Puttisong, Irina Buyanova, S. Suraprapapich, C. W. Tu and Weimin Chen, Temperature dependence of dynamic nuclear polarization and its effect on electron spin relaxation and dephasing in InAs/GaAs quantum dots, 2012, Applied Physics Letters, (100), 14, 143105.

http://dx.doi.org/10.1063/1.3701273

Copyright: American Institute of Physics (AIP) http://www.aip.org/

Postprint available at: Linköping University Electronic Press http://urn.kb.se/resolve?urn=urn:nbn:se:liu:diva-75095 


\title{
Temperature dependence of dynamic nuclear polarization and its effect on electron spin relaxation and dephasing in InAs/GaAs quantum dots
}

\author{
J. Beyer, ${ }^{1}$ Y. Puttisong, ${ }^{1}$ I. A. Buyanova, ${ }^{1}$ S. Suraprapapich, ${ }^{2}$ C. W. Tu, ${ }^{2}$ and W. M. Chen ${ }^{1}$ \\ ${ }^{1}$ Department of Physics, Chemistry and Biology, Linköping University, SE-581 83 Linköping, Sweden \\ ${ }^{2}$ Department of Electrical and Computer Engineering, University of California, La Jolla, California 92093 , \\ USA
}

(Received 10 February 2012; accepted 18 March 2012; published online 3 April 2012)

\begin{abstract}
Electron spin dephasing and relaxation due to hyperfine interaction with nuclear spins is studied in an InAs/GaAs quantum dot ensemble as a function of temperature up to $85 \mathrm{~K}$, in an applied longitudinal magnetic field. The extent of hyperfine-induced dephasing is found to decrease, whereas dynamic nuclear polarization increases with increasing temperature. We attribute both effects to an accelerating electron spin relaxation through phonon-assisted electron-nuclear spin flip-flops driven by hyperfine interactions, which could become the dominating contribution to electron spin depolarization at high temperatures. (C) 2012 American Institute of Physics. [http://dx.doi.org/10.1063/1.3701273]
\end{abstract}

Carrier spins in semiconductor quantum dots (QDs) are an area of intense current research interest due to their potential applications in spintronics, ranging from spin-light-emitting-diodes (spin-LEDs) (Ref. 1) to quantum computation. ${ }^{2}$ The main sources of spin depolarization in QDs at low temperatures have been identified as the exchange interaction between unpaired carrier spins $s^{3,4}$ and the hyperfine interaction with the nuclear spin ensemble of the lattice atoms. ${ }^{5-7}$ Due to stronger hyperfine coupling between electron and nuclear spins in a confined QD system, transfer of nonequilibrium spins between electrons and nuclei becomes increasingly important as compared with unconfined systems, resulting in efficient dynamic nuclear polarization (DNP) ${ }^{8-11}$ This has led to the proposal of transferring quantum information from the electron spin state to a long-living nuclear spin state for quantum information storage. ${ }^{12}$ The effective magnetic field of the DNP, on the other hand, will act back on the electron spin by suppressing its dephasing in the random correlations of the QD nuclear spin fluctuations (NSFs), thereby extending the electron spin lifetime. ${ }^{13}$ At the same time, the DNP field will split the electron spin levels, which restricts a further DNP build-up due to the energy mismatch with the negligible nuclear spin level splitting. This effect limits the attainable DNP degree at low temperatures. Only recently, a study of the aspect of temperature dependence of DNP degree in single, positively charged InGaAs QDs has been reported. ${ }^{11}$ In a rather strong magnetic field of $2 \mathrm{~T}$, the efficiency of DNP build-up was found to increase with lattice temperature up to $55 \mathrm{~K}$, which was attributed to a broadening of the Zeeman-split spin levels. This eases the limitation on further DNP build-up. A similar increase in DNP efficiency was concluded in an n-doped IIVI QD ensemble for temperatures up to $100 \mathrm{~K} .{ }^{14} \mathrm{Up}$ to now, however, there are still many open questions to be answered that are of both fundamental scientific interest and technological relevance. They include, e.g., how DNP will develop with a further increase in temperature, whether the hyperfine interactions will remain as a dominant mechanism for electron spin relaxation and dephasing at higher temperatures, and what the dominant physical mechanism determining electron spin polarization and coherence at room temperature is. In this letter, we aimed to investigate the temperature dependence of DNP and electron spin relaxation/dephasing in an ensemble of positively charged InAs QDs until reaching the temperature limit when they are no longer accessible in our experiments. For this, we carried out a detailed study of electron spin polarization of positive trions in an external longitudinal magnetic field as a function of temperature, which has allowed us to simultaneously examine the DNP generation efficiency and the extent of hyperfine-induced spin dephasing and relaxation.

The measurements were conducted on a sample of selfassembled InAs QDs, grown by molecular beam epitaxy (MBE) on a (001) semi-insulating GaAs substrate in the Stranski-Krastanov growth mode. Deposition of 1.8 monolayers (ML) InAs at $500^{\circ} \mathrm{C}$ on a $300 \mathrm{~nm}$ GaAs buffer layer resulted in large QDs residing on a thin InGaAs wetting layer (WL). A typical dot height is around $12 \mathrm{~nm}$, and their diameter $40-60 \mathrm{~nm}$. Details of the growth procedure may be found in Ref. 15. Optical orientation spectroscopy in a longitudinal magnetic field was employed under non-resonant excitation above the GaAs barrier. The circular polarization of the excitation light from a Ti:Sapphire laser was controlled either by a rotatable broad-band quarter-wave plate, allowing continuous-wave excitation of a fixed helicity, either $\sigma^{+}$or $\sigma^{-}(\mathrm{cw})$, or by a photoelastic modulator (PEM), which provided excitation with an alternating helicity between $\sigma^{+}$ and $\sigma^{-}$at a frequency of $50 \mathrm{kHz}$. Polarization of the resulting photoluminescence (PL) from the QD ground state was resolved either by a PEM or by a rotatable broadband quarter-wave plate in conjunction with a linear polarizer and detected by a liquid nitrogen-cooled Ge-diode connected to a monochromator. The samples were kept in a liquid helium flow cryostat and placed in the magnetic field of an electromagnet, oriented along the sample normal. Optical excitation and detection were conducted along the same direction, i.e., in a Faraday geometry. Due to residual p-type doping introduced during the MBE growth process, positive trions $\mathrm{X}^{+}$were observed in a majority of the QDs upon capture of an optically excited electron-hole-pair. Their circular polarization degree 
$P=-\rho$ provides a direct access to the spin polarization of the electrons $\rho$, as the two hole spins are paired off in the $\mathrm{X}^{+}$. Also the exchange interaction cancels out in the $\mathrm{X}^{+}$ground state, leaving the hyperfine interaction with the QD nuclear spins as the main mechanism of electron spin depolarization.

The electron spin polarization degree is affected by spin dephasing in random fluctuations of the nuclear spin system $^{5-7}$ (NSFs) and also by transfer of the electron's spin angular momentum to the nuclear spin system, leading to the build-up of a DNP field ${ }^{8,10} B_{\mathrm{N}}$ (often referred to as an Overhauser field). The electron spin dephasing mechanism can be suppressed in an external longitudinal magnetic field $B_{\mathrm{Z}}$ if it is stronger than the transverse effective field of the NSF $\left(\delta B_{\mathrm{N}}\right){ }^{5,7,10}$ The resulting increase in spin polarization and, thus, PL polarization degree $P$ in the QD ground state as a function of $B_{\mathrm{z}}$ can be described by a Lorentzian line, ${ }^{10}$

$$
P\left(B_{\mathrm{z}}\right)=P(\infty)\left(1-\frac{A_{\mathrm{dip}}}{1+\left(\left(B_{\mathrm{z}}+B_{\mathrm{N}}\right) / \delta B_{\mathrm{N}}\right)^{2}}\right) .
$$

Here, $P(\infty)$ is the PL polarization degree in strong magnetic fields, when the NSF dephasing is completely suppressed. $A_{\text {dip }}$ is the depth of the polarization dip characterizing the extent of the spin dephasing by the NSF, when the total magnetic field $B_{\mathrm{Z}}+B_{\mathrm{N}}=0$. Therefore, the position, width, and depth of the polarization dip should provide us with the means to study properties of the DNP, the NSF-induced spin dephasing, and the effect of DNP on spin relaxation.

In Fig. 1(a), a $P\left(B_{\mathrm{z}}\right)$ curve at $6 \mathrm{~K}$ is shown, obtained under alternating $\sigma^{+}$and $\sigma^{-}$excitation by a PEM and also
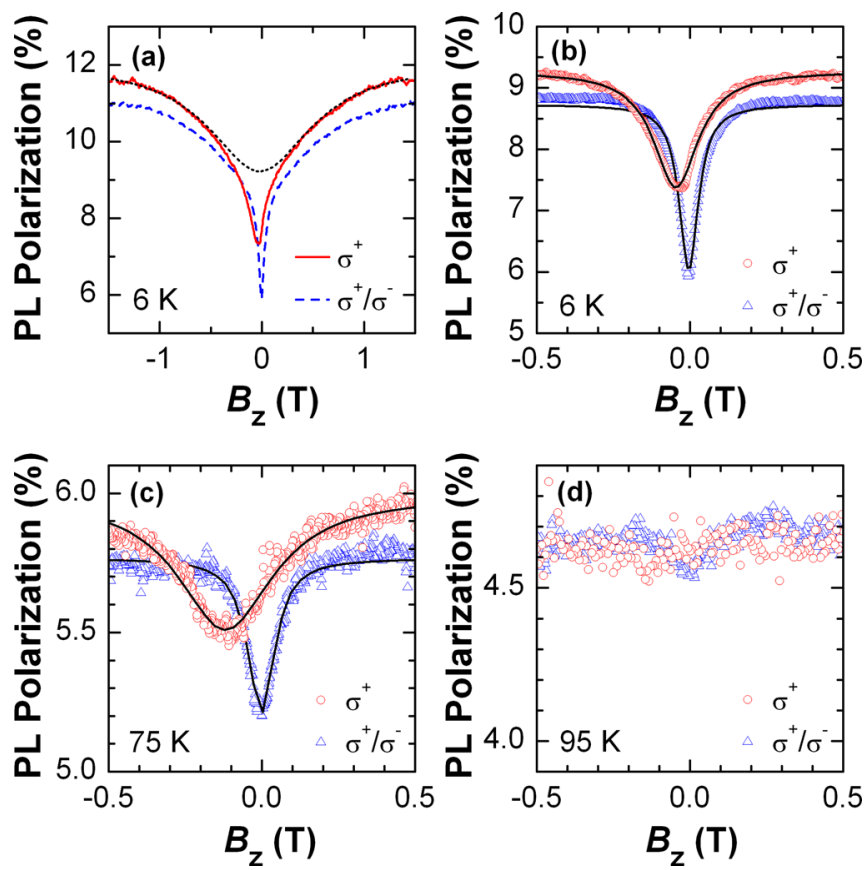

FIG. 1. The circular polarization degree of the QD ground state as a function of an external longitudinal magnetic field $B_{\mathrm{z}}$ under $\mathrm{cw} \sigma^{+}$and alternating $\sigma^{+}$and $\sigma^{-}$excitation. (a) The data over a wide magnetic field range at $6 \mathrm{~K}$. The black dotted line is the broad component, attributed to suppression of AEI in $\mathrm{X}^{0}$. (b) The sharp components with their corresponding Lorentzian fits in a close-up where the broad component has been removed for clarity. (c) and (d) The corresponding data at $75 \mathrm{~K}$ and $95 \mathrm{~K}$. under $\mathrm{cw} \sigma^{+}$excitation. A sharp polarization dip in weak magnetic fields and a considerably wider dip, shown by the dotted line in Fig. 1(a), were observed under both excitation conditions. The wider dip component does not exhibit an Overhauser shift and remains the same lineshape independent of the excitation conditions (i.e., $\mathrm{cw} \sigma^{+}$or PEM) and temperature. It can be attributed to the suppression of the anisotropic exchange interaction (AEI) in neutral QD excitons ${ }^{4,16}$ that are also present in our structure. As it is unrelated to DNP and is beyond the scope of the present work, this wide component will not be discussed further below. For easier viewing of the sharp component arising from the positive trions, we have subtracted the wide component from the data in Figs. 1(b)-1(d).

In strong contrast, the sharp component is found to be sensitive to both excitation protocol and temperature. The dip position of this component shifts away from zero magnetic field under $\mathrm{cw} \sigma^{+}$excitation, corresponding to an Overhauser field $B_{\mathrm{N}} \approx 45 \mathrm{mT}$ due to DNP, as shown by the open circles in Fig. 1(b). As the DNP build-up time is known to be in the order of $\mathrm{ms},{ }^{17}$ no DNP is expected under alternating $\sigma^{+}$and $\sigma^{-}$excitation at $50 \mathrm{kHz}$ by a PEM as confirmed by our experimental results (the open triangles in Fig. 1(b)). This characteristic provides identification of the origin of the sharp component as being related to hyperfine induced DNP and the dephasing of the trion electron in the NSF field. ${ }^{5,18}$

From a best fit of Eq. (1) to the data, we extract $\delta B_{\mathrm{N}}^{\mathrm{PEM}} \approx 40 \mathrm{mT}$ and $A_{\mathrm{dip}}^{\mathrm{PEM}}=0.3$ under PEM excitation. The deduced $\delta B_{\mathrm{N}}^{\mathrm{PEM}}$ is in the range of previous reports. ${ }^{10,19}$ Under $\mathrm{cw} \sigma^{+}$excitation, on the other hand, a significant broadening of the dip, $\delta B_{\mathrm{N}}^{\mathrm{cw}} \approx 90 \mathrm{mT}$, and a much shallower depth with $A_{\text {dip }}^{\mathrm{cw}}=0.2$ are observed.

To assure that the position and width of the dip under the PEM excitation represent the true state of the QDs without any DNP field and that these are not due to an averaging over two opposite DNP fields individually built up during the alternating $\sigma^{+}$and $\sigma^{-}$excitation, we carried out a detailed study of the DNP field by resolving the PL polarization degree as a function of $B_{\mathrm{z}}$ over time. The results are shown in Fig. 2. The alternating $\sigma^{+}$and $\sigma^{-}$excitation at

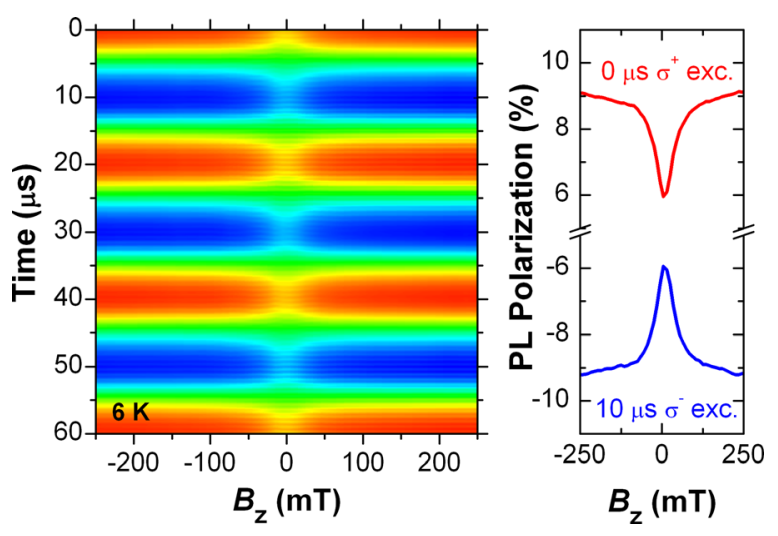

FIG. 2. The left panel shows a 2D-plot of the QD PL polarization degree, as a function of time and $B_{\mathrm{z}}$ under alternating $\sigma^{+}$and $\sigma^{-}$excitation by a PEM. The red and blue colors correspond to positive and negative circular polarization degree, respectively. The right panel shows the horizontal crosssections of the 2D-plot at two given times, when the excitation polarization is $\sigma^{+}$(upper trace, at $0 \mu \mathrm{s}$ ) and $\sigma^{-}$(lower trace, at $10 \mu \mathrm{s}$ ). 
$50 \mathrm{kHz}$ leads to the observed periodic pattern of the PL polarization. Here, the red and blue colors signify positive and negative circular polarization degrees of the PL, respectively. It can be seen that the polarization dip, corresponding to the lighter red for $\sigma^{+}$and the lighter blue for $\sigma^{-}$polarization degrees, is fixed at $B_{\mathrm{z}}=0$ throughout the entire excitation period. In the right panel of Fig. 2, two cross-sections of the $2 \mathrm{D}$ plot are shown, taken at the times that correspond to $\sigma^{+}(0 \mu \mathrm{s})$ and $\sigma^{-}(10 \mu \mathrm{s})$ excitation. Clearly, there is no shift of the polarization dip away from zero field, which confirms that there is no build-up of any noticeable $B_{\mathrm{N}}$ during the approximately $10 \mu \mathrm{s}$ of excitation with the same helicity. This finding also rules out the possibility that the polarization dip width under alternating helicity excitation is due to a time-integration of varying $B_{\mathrm{N}}$ over time. We can thus conclude that the sharp dip shown in Fig. 1(b) is a true measure of the NSF strength $\delta B_{\mathrm{N}}$ of the QD ensemble. We attribute the observed larger dip width $\delta B_{\mathrm{N}}^{\mathrm{cw}}$ under the cw $\sigma^{+}$excitation to a spread of the DNP field values in the inhomogeneous QD ensemble. ${ }^{10}$ This spread will also prevent the external field $B_{\mathrm{z}}$ from cancelling the DNP for all QDs in the ensemble at once. This may contribute to the observed decrease in dip depth under $\mathrm{cw} \sigma^{+}$conditions.

To examine the importance of the hyperfine induced spin dephasing and relaxation at high temperatures, we have conducted detailed investigations of the trion's electron spin polarization as a function of temperature. Fig. 1(c) shows the results obtained at $75 \mathrm{~K}$. Under PEM excitation, no DNP field can be observed and $\delta B_{\mathrm{N}}^{\mathrm{PEM}}$ is found to be roughly the same as that at $6 \mathrm{~K}$. Under $\mathrm{cw} \sigma^{+}$excitation, however, the DNP field increases to $B_{\mathrm{N}} \approx 110 \mathrm{mT}$. At the same time, the dip broadens further and becomes even shallower. At above $85 \mathrm{~K}$, it can no longer be observed, as shown in Fig. 1(d).

In Fig. 3(a), we plot the characteristic quantities of the polarization dip as a function of temperature until it disappears at $T>85 \mathrm{~K}$. It can be clearly seen that $\delta B_{\mathrm{N}}^{\mathrm{PEM}}$ is indeed temperature independent. This is reasonable as it arises from random correlations in the NSF, for which no temperature dependence is expected. In contrast, both $B_{\mathrm{N}}$ and $\delta B_{\mathrm{N}}^{\mathrm{cw}}$ increase continuously with increasing temperature. The average $B_{\mathrm{N}}$ increases from just above $40 \mathrm{mT}$ at $6 \mathrm{~K}$ to around $110 \mathrm{mT}$ at $85 \mathrm{~K}$. The close correlation between $\delta B_{\mathrm{N}}^{\mathrm{cw}}$ and $B_{\mathrm{N}}$ supports our interpretation that the increase of $\delta B_{\mathrm{N}}^{\mathrm{cw}}$ is due to an increasing spread of the DNP field in the QD ensemble. To further verify the correlation between $\delta B_{\mathrm{N}}^{\mathrm{cw}}$ and $B_{\mathrm{N}}$, we performed similar experiments at a fixed temperature by varying excitation power, bearing in mind that increasing excitation density is expected to enhance DNP. The results obtained at $6 \mathrm{~K}$ are shown in Fig. 3(b). They confirm that a stronger DNP field is accompanied by a broader dip width of the spin polarization curve in a longitudinal field.

The fact that the polarization dip weakens with increasing temperature and is no longer observable at temperatures higher than $85 \mathrm{~K}$, as shown in the lower panel of Fig. 3(a), indicates a reducing effect of the electron spin dephasing by the NSF with increasing temperature. This is valid under both cw and PEM excitation. For example, the NSF-induced spin dephasing reduces spin polarization by about $30 \%$ at $6 \mathrm{~K}$, but the corresponding value at $85 \mathrm{~K}$ is only about $5 \%$. The accompanying reduction in the overall degree of spin


FIG. 3. (a) Upper panel: Dynamically created nuclear field $B_{\mathrm{N}}$ and width of the polarization dip under $\mathrm{cW} \sigma^{+}\left(\delta B_{\mathrm{N}}^{\mathrm{cw}}\right)$ and PEM $\left(\delta B_{\mathrm{N}}^{\mathrm{PEM}}\right)$ excitation as a function of sample temperature. Lower panel: Dip depth under $\mathrm{cw} \sigma^{+}$excitation $A_{\text {dip }}^{\mathrm{cW}}$ and under PEM excitation $A_{\mathrm{dip}}^{\mathrm{PEM}}$ as a function of sample temperature. (b) $B_{\mathrm{N}}, \delta B_{\mathrm{N}}^{\mathrm{cw}}$, and $\delta B_{\mathrm{N}}^{\mathrm{PEM}}$ as a function of excitation power density, here given as PL signal strength, at $6 \mathrm{~K}$.

polarization over the studied temperature range, as seen from Fig. 1, suggests that other spin depolarization mechanisms have significantly gained importance. The observed increasing DNP field with increasing temperature provides the evidence for such a mechanism, i.e., electron spin relaxation mediated by the hyperfine induced electron-nuclear spin flipflops- the same process driving the DNP. Such spin relaxation can be accelerated at higher temperatures, because the bottleneck of the flip-flop process at low temperatures-the energy mismatch in spin splittings between the electron and the nuclei-can now be overcome by thermal broadening of these spin levels. ${ }^{11}$ The enhanced spin relaxation can interrupt and strongly suppress the spin dephasing by the NSF when the former is much faster than the latter. Our finding thus suggests that the phonon-assisted hyperfine-induced electron spin relaxation is a possible candidate for the dominant spin depolarization process that controls electron spin polarization at $85 \mathrm{~K}$ and likely at even higher temperatures.

In conclusion, we have been able to characterize the hyperfine-induced electron spin dephasing and relaxation as well as the DNP generation efficiency in an ensemble of InAs/GaAs QDs over the entire temperature range (up to $85 \mathrm{~K}$ ) when they are accessible in our experiments. From their temperature dependence, we conclude that, while the spin dephasing in the NSF decreases with increasing temperature and becomes negligible at $85 \mathrm{~K}$, the hyperfine induced 
electron spin relaxation gains importance and is suggested to eventually control the spin polarization at $85 \mathrm{~K}$. We believe that the conclusion on the negligible spin dephasing should still hold even at $T>85 \mathrm{~K}$. However, no definite conclusion on the dominance of the hyperfine-induced spin relaxation at $T>85 \mathrm{~K}$, though probable, can be drawn at present. Further studies are required to clarify this issue.

We gratefully acknowledge The Swedish Research Council (Grant No. 621-2011-4254) for financial support.

${ }^{1}$ M. Holub and P. Bhattacharya, J. Phys. D: Appl. Phys. 40, R179 (2007).

${ }^{2}$ D. Loss and D. P. DiVincenzo, Phys. Rev. A 57, 120 (1998); V. Cerletti, W. A. Coish, O. Gywat, and D. Loss, Nanotechnology 16, R27 (2005).

${ }^{3}$ D. Gammon, E. S. Snow, B. V. Shanabrook, D. S. Katzer, and D. Park, Phys. Rev. Lett. 76, 3005 (1996); R. Dzhioev, B. Zakharchenya, E. Ivchenko, V. Korenev, Yu. Kusraev, N. Ledentsov, V. Ustinov, A. Zhukov, and A. Tsatsul'nikov, Phys. Solid State 40, 790 (1998); M. Bayer, A. Kuther, A. Forchel, A. Gorbunov, V. B. Timofeev, F. Schäfer, J. P. Reithmaier, T. L. Reinecke, and S. N. Walck, Phys. Rev. Lett. 82, 1748 (1999).

${ }^{4}$ M. Sénès, B. Urbaszek, X. Marie, T. Amand, J. Tribollet, F. Bernardot, C. Testelin, M. Chamarro, and J. M. Gèrard, Phys. Rev. B 71, 115334 (2005).

${ }^{5}$ I. A. Merkulov, Al. L. Efros, and M. Rosen, Phys. Rev. B 65, 205309 (2002).

${ }^{6}$ A. V. Khaetskii, D. Loss, and L. Glazman, Phys. Rev. Lett. 88, 186802 (2002); J. R. Petta, A. C. Johnson, J. M. Taylor, E. A. Laird, A. Yacoby, M. D. Lukin, C. M. Marcus, M. P. Hanson, and A. C. Gossard, Science 309, 2180 (2005).

${ }^{7}$ P. F. Braun, X. Marie, L. Lombez, B. Urbaszek, T. Amand, P. Renucci, V. K. Kalevich, K. V. Kavokin, O. Krebs, P. Voisin, and Y. Masumoto, Phys. Rev. Lett. 94, 116601 (2005).

${ }^{8}$ V. K. Kalevich, K. V. Kavokin, and I. A. Merkulov, in Spin Physics in Semiconductors, edited by M. I. Dyakonov (Springer, Berlin, 2008), Vol.
157, Chap. 11; B. Eble, O. Krebs, A. Lemaitre, K. Kowalik, A. Kudelski, P. Voisin, B. Urbaszek, X. Marie, and T. Amand, Phys. Rev. B 74, 081306 (2006); I. A. Akimov, D. H. Feng, and F. Henneberger, Phys. Rev. Lett. 97(5), 056602 (2006); S. W. Brown, T. A. Kennedy, D. Gammon, and E. S. Snow, Phys. Rev. B 54, 17339 (1996); R. I. Dzhioev, B. P. Zakharchenya, V. L. Korenev, P. E. Pak, M. N. Tkachuk, D. A. Vinokurov, and I. S. Tarasov, JETP Lett. 68, 745 (1998); C. W. Lai, P. Maletinsky, A. Badolato, and A. Imamoglu, Phys. Rev. Lett. 96, 167403 (2006).

${ }^{9}$ D. Gammon, A. L. Efros, T. A. Kennedy, M. Rosen, D. S. Katzer, D. Park, S. W. Brown, V. L. Korenev, and I. A. Merkulov, Phys. Rev. Lett. 86, 5176 (2001); E. A. Chekhovich, A. B. Krysa, M. S. Skolnick, and A. I. Tartakovskii, Phys. Rev. B 83, 125318 (2011).

${ }^{10}$ R. V. Cherbunin, S. Yu Verbin, T. Auer, D. R. Yakovlev, D. Reuter, A. D. Wieck, I. Ya Gerlovin, I. V. Ignatiev, D. V. Vishnevsky, and M. Bayer, Phys. Rev. B 80, 035326 (2009).

${ }^{11}$ B. Urbaszek, P. F. Braun, T. Amand, O. Krebs, T. Belhadj, A. Lemaître, P. Voisin, and X. Marie, Phys. Rev. B 76, 201301 (2007).

${ }^{12}$ J. M. Taylor, C. M. Marcus, and M. D. Lukin, Phys. Rev. Lett. 90, 206803 (2003).

${ }^{13}$ R. Oulton, A. Greilich, S. Y. Verbin, R. V. Cherbunin, T. Auer, D. R. Yakovlev, M. Bayer, I. A. Merkulov, V. Stavarache, D. Reuter, and A. D. Wieck, Phys. Rev. Lett. 98, 107401 (2007).

${ }^{14}$ V. Gapon, J. Puls, and F. Henneberger, Appl. Phys. Lett. 94, 213111 (2009).

${ }^{15}$ S. Suraprapapich, S. Panyakeow, and C. W. Tu, Appl. Phys. Lett. 90, 183112 (2007); S. Suraprapapich, Y. M. Shen, V. A. Odnoblyudov, Y. Fainman, S. Panyakeow, and C. W. Tu, J. Cryst. Growth 301-302, 735 (2007).

${ }^{16}$ M. Bayer, G. Ortner, O. Stern, A. Kuther, A. A. Gorbunov, A. Forchel, P. Hawrylak, S. Fafard, K. Hinzer, T. L. Reinecke, S. N. Walck, J. P. Reithmaier, F. Klopf, and F. Schäfer, Phys. Rev. B 65, 195315 (2002).

${ }^{17}$ P. Maletinsky, A. Badolato, and A. Imamoglu, Phys. Rev. Lett. 99, 056804 (2007).

${ }^{18}$ M. Yu Petrov, I. V. Ignatiev, S. V. Poltavtsev, A. Greilich, A. Bauschulte, D. R. Yakovlev, and M. Bayer, Phys. Rev. B 78, 045315 (2008).

${ }^{19}$ O. Krebs, B. Eble, A. Lemaitre, B. Urbaszek, K. Kowalik, A. Kudelski, X. Marie, T. Amand, and P. Voisin, Phys. Status Solidi A 204, 202 (2007). 